\title{
Is there a correlation between upper lumbar disc herniation and multifidus muscle degeneration? A retrospective study of MRI morphology
}

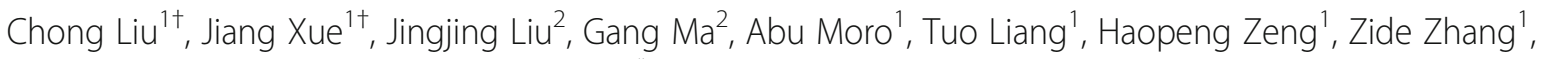
Guoyong $X u^{1}$, Zhaojun $L^{1}$ and Xinli Zhan ${ }^{1 *}$ (D)

\begin{abstract}
Background: The purpose of the study is to investigate the correlation between upper lumbar disc herniation $(U L D H)$ and multifidus muscle degeneration via the comparison of width, the cross-sectional area and degree of fatty infiltration of the lumbar multifidus muscle.

Methods: Using the axial T2-weighted images of magnetic resonance imaging as an assessment tool, we retrospectively investigated 132 patients with ULDH and 132 healthy individuals. The total muscle cross-sectional area (TMCSA) and the pure muscle cross-sectional area (PMCSA) of the multifidus muscle at the L1/2, L2/3, and L3/4 intervertebral disc levels were measured respectively, and in the meantime, the average multifidus muscle width (AMMW) and degree of fatty infiltration of bilateral multifidus muscle were evaluated. The resulting data were analyzed to determine the presence/absence of statistical significance between the study and control groups. Multivariate logistical regression analyses were used to evaluate the correlation between ULDH and multifidus degeneration.

Results: The results of the analysis of the two groups showed that there were statistically significant differences $(p<0.05)$ between TMCSA, PMCSA, AMMW and degree of fatty infiltration. The multivariate logistic regression analysis indicated that the TMCSA, PMCSA, AMMW and the degree of fatty infiltration of multifidus muscle were correlated with ULDH, and the differences were statistically significant $(P<0.05)$.

Conclusions: A correlation could exist between multifidus muscles degeneration and ULDH, that may be a process of mutual influence and interaction. Lumbar muscle strengthening training could prevent and improve muscle atrophy and degeneration.
\end{abstract}

Keywords: Upper lumbar disc herniation, Multifidus muscle, Muscle degeneration, Fatty infiltration

* Correspondence: zhan191823@163.com

Chong Liu and Jiang Xue were both the first author.

'Spine and Osteopathy Ward, The First Affiliated Hospital of Guangxi Medical University, No.6 Shuangyong Road, 530021 Nanning, Guangxi, People's Republic of China

Full list of author information is available at the end of the article

\section{Background}

Lumbar disc herniation (LDH) is a primary cause of lower back pain and sciatica in adults. Generally, in most patients the involvement of the lumbar segments L4/5 and L5/S1 are known to be associated with radicular pain, sensory deficits, or motor weakness [1]. Disc herniation at the L3/4 and above are referred to as upper

(c) The Author(s). 2021 Open Access This article is licensed under a Creative Commons Attribution 4.0 International License, which permits use, sharing, adaptation, distribution and reproduction in any medium or format, as long as you give appropriate credit to the original author(s) and the source, provide a link to the Creative Commons licence, and indicate if changes were made. The images or other third party material in this article are included in the article's Creative Commons licence, unless indicated otherwise in a credit line to the material. If material is not included in the article's Creative Commons licence and your intended use is not permitted by statutory regulation or exceeds the permitted use, you will need to obtain permission directly from the copyright holder. To view a copy of this licence, visit http://creativecommons.org/licenses/by/4.0/ The Creative Commons Public Domain Dedication waiver (http://creativecommons.org/publicdomain/zero/1.0/) applies to the data made available in this article, unless otherwise stated in a credit line to the data. 
lumbar disc herniation (ULDH), these account for under $10 \%$ of lumbar disc herniations $[2,3]$. The precise pathogenesis of disc herniation is yet to be established fully. However, most researchers believe it to be closely related to the anatomical characteristics [3-6].

Under normal physiological conditions, the various tissues and structures of the spine can maintain physiological stability, which prevents the spinal cord and spinal nerve roots from getting compressed or damaged [7]. The muscles that act locally to stabilize the spine include the transverse abdominis, psoas major, and multifidus muscles, and the functions of these muscles include controlling the spinal curvature and maintaining its mechanical stability. The multifidus muscles are the most important muscles for local stabilizing $[8,9]$. The lumbar multifidus muscle (LMM) is the largest group of posterior muscles in the lumbosacral region [10]. The LMM specifically acts to maintain segmental stability of the spine, maintain lumbar physiological lordosis, control facet joint movement, and adjust the distribution of the intervertebral load and pressure [11]. However, unlike the other paraspinal muscles, the multifidus muscles receive only a unilateral single-segmental innervation $[11,12]$. Considering the crucial roles fulfilled by the multifidus muscle for maintaining spinal stability, a decline in its physiological functions could result in changing to the original biomechanical relationship.

Recently, studies have indicated that a causal relationship may exist between LDH and multifidus muscle degeneration [13]. Compared to healthy individuals, patients with LDH have a higher degree of fatty infiltration of the multifidus muscle and smaller muscle cross-sectional area. Moreover, the associated radiological features of paravertebral soft tissue involvements, muscle degeneration and fatty infiltration were reported in LDH patients [14-17]. Reportedly, fatty infiltration of the multifidus muscle was closely related to LDH; however, such an association was not significant in adolescents [18]. Most past studies of the LMM and LDH have primarily focused on the L4/5 and L5/S1 segments [4-6, 14]. However, to the best knowledge of the authors, only a few studies have explored the relationship between multifidus muscle degeneration and ULDH. It has been demonstrated that the indirect evaluation of the features of the multifidus muscle by measuring the intensity and range of magnetic resonance imaging (MRI) signals are both effective and reliable [14, 17, 19]. This study compares the MRI features of LMM, which includes the muscle cross-sectional area, width and the degree of fatty infiltration between ULDH patients and healthy individuals, to explore the association between ULDH and the changes of the LMM.

\section{Methods}

\section{Study population}

Patients with single segment ULDH (herniation of the $\mathrm{L} 1 / 2, \mathrm{~L} 2 / 3$, or $\mathrm{L} 3 / 4$ ) who received surgical treatment from 2012 to 2019 in the First Affiliated Hospital of Guangxi Medical University (Nanning, China) were selected as the study subjects. The diagnostic criteria are as follows: Firstly, MRI scans indicated the presence of LDH and surrounding soft tissue involvement, including the presence of compressed nerves, which constitutes a necessary diagnostic criterion. Secondly, patients with LDH present signs and symptoms consistent with the area of innervation of the affected nerve, such as radicular lower limb pain, paraesthesia, muscle weakness, diminished tendon reflexes, positive straight leg raising test/femoral stretch test, etc. It is common for LDH patients to exhibit at least three of these manifestations. Moreover, the presence of polyradiculopathies caused by a single disc herniation at higher levels requires a confirmatory diagnosis that combining clinical features and MRI. The exclusion criteria are as follows: Firstly, patients with confirmed MRI diagnosis of lower lumbar disc herniations, including L4-L5 or L5-S1 segments. Secondly, patients with other pathologies, including lumbar spinal stenosis, lumbar spondylolisthesis, scoliosis, spinal fracture, spinal tuberculosis, spinal tumours, etc. Thirdly, patients with pre-existing cardiovascular, pulmonary, cerebrovascular, and neuromuscular disorders that could lead to motor dysfunctions were excluded. Lastly, patients with a history of lumbar surgery or longterm lower back pain were also excluded. Additionally, incomplete data and poor-quality MRI images were also not included.

The control group included healthy subjects who underwent MRI examination at the outpatient department or physical examination centre of the hospital where the study was conducted, they had no history of disc herniation or lumbar degenerative diseases. The exclusion criteria include people with tuberculosis, tumours, fractures, spinal deformities, LDH, lumbar spondylolisthesis, lumbar spinal stenosis, and other lumbar spine diseases, or those with poor MR image quality that were immeasurable. The age group of all participants ranged between 20 and 65 years.

During the study period, 245 patients with ULDH were hospitalized. Under the inclusion and exclusion screening, a total of 132 patients were eligible to enter the study group (including 82 males and 50 females). The control group included healthy individuals with complete physical examination data were randomly selected for the case-control study. The collected data included age, gender, body mass index (BMI), occupation, and lumbar MRI scans for intergroup statistical comparisons. Due to random selection, there is no significant 
difference in the sex ratio, occupational composition and segments of the intervertebral disc between the study group and the control group.

This study was approved by the Ethics Committee of the First Affiliated Hospital of Guangxi Medical University, and obtained permissions to access clinical/personal patient data used in our research. Written informed consent was obtained from all of the participants in the study.

\section{Image acquisition and data analysis}

The MRI data of all subjects reviewed for this study were acquired from the same MRI device (3.0 T; Magnetom Verio; Siemens Medical Solutions, Germany). And performing scans based on the following parameters: the axial T2-weighted images from L1 to S1 (TR/TE 3000/ 110 , matrix size $256 \times 256$, time to recovery: $3,000-3$, $600 \mathrm{~ms}$, time to echo: $80-105 \mathrm{~ms}$, and slice thickness: 4 $\mathrm{mm})$. The axial T2-weighted images of the MRI of all subjects were collected and analysed using the Picture Archiving and Communication Systems (version 2.7.1.0; PACS Clinical Viewer ANTAI; CHINA). All the obtained MRI scans of the study group were performed before the patients received surgical treatment. In the study group, a segmental plane of a herniated lumbar disc (L1/2, L2/3, or L3/4) of the axial T2-weighted images was measured at the mid-disc. The same plane of the control group was measured using data matching. All measurements were taken by a qualified radiologist with several years of experience using blinding, and measurement software. The total cross-sectional area of the muscle and the interlaminar fat were measured along the edge of the multifidus muscle (Fig. 1A, B). The PACS software was used to identify the boundary of multifidus muscle and fat in the axial images. The pure cross-sectional area of the multifidus muscle (Fig. 1C)

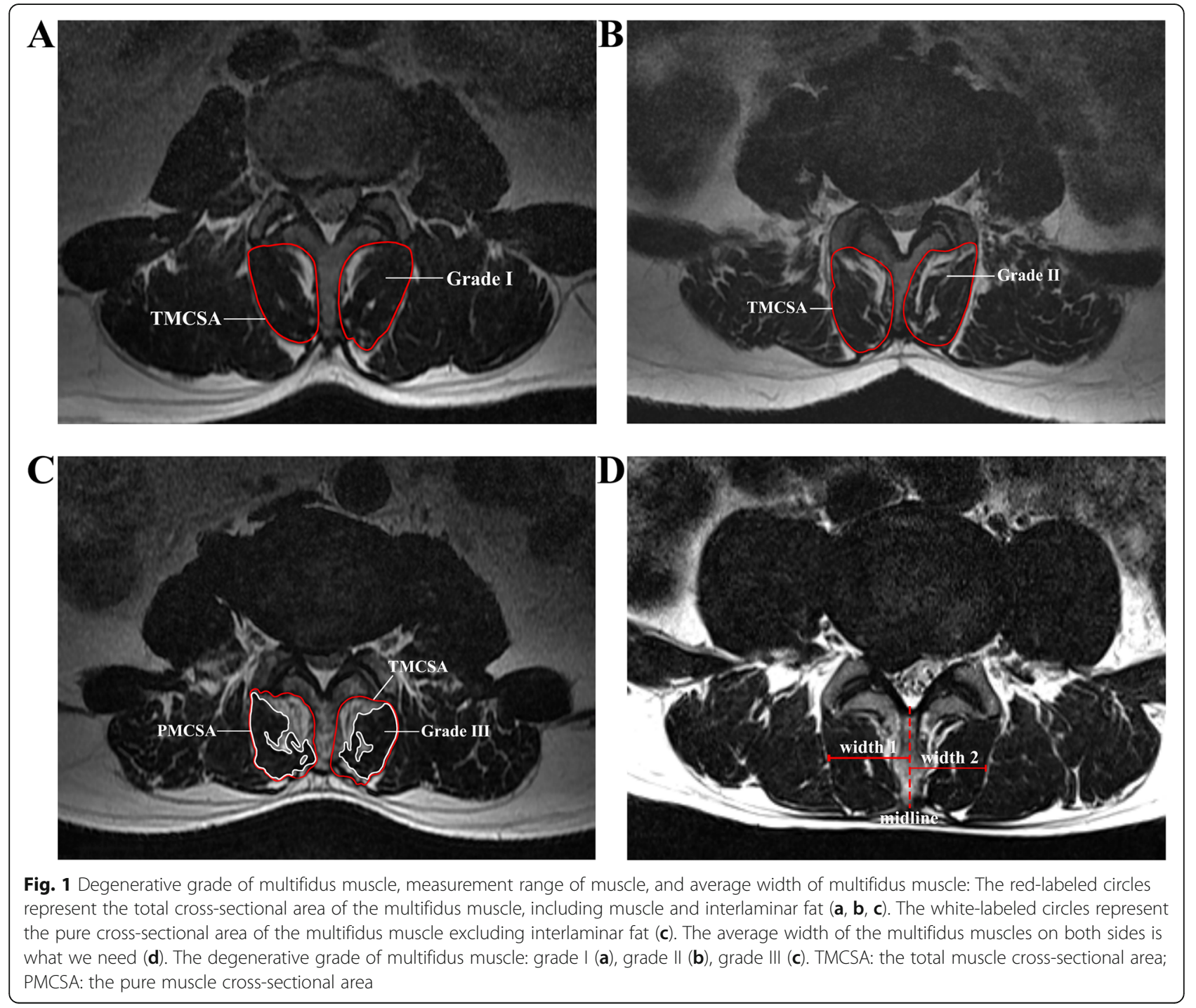


was measured by excluding fat signal from the segmentation. The horizontal distance from the most prominent point of the outer margin of the multifidus muscle in the disc plane to the midline of the spinous process was measured to evaluate the multifidus muscle width (Fig. 1D). Based on the theoretical and practical studies of Battaglia et al.[20] and Ekin et al.[21], the visual difference between the amount of fatty infiltration was used to evaluate and grade the degree of fatty infiltration of the LMM:

Grade I: Normal muscle or mild fat infiltration, with dotted or linear infiltrated areas, fatty infiltration $<10 \%$ (Fig. 1A).

Grade II: Moderate fat infiltration, with multiple patchy infiltrations areas, fatty infiltration 10\%-50\% (Fig. 1B).

Grade III: Severe fatty infiltration, exhibiting feathery or mesh-like infiltrations areas; fatty infiltration $>=50 \%$ (Fig. 1C).

Statistical analyses were performed using the SPSS software (version 25.0; SPSS IBM; USA). The Chisquared test was adopted to analyse the differences in age groupings, BMI distribution, and degree of fatty infiltration between the herniation group and the control group. The total muscle cross-sectional area (TMCSA), pure muscle cross-sectional area (PMCSA), average multifidus muscle width (AMMW), and other measurement data were measured three times, and the average of four measured values was expressed as mean \pm standard deviation. The ANOVA was used to analyse and compare the differences in TMCSA, PMCSA, and AMMW between the two group. Multivariate logistic regression analysis was used to analyse the correlation between the ULDH and the factors, such as age, BMI, TMCSA, PMCSA, AMMW and the degree of fatty infiltration. $P<0.05$ indicates the presence of statistically significant differences.

\section{Results}

This study included a total of 264 participants, including 132 in the study group and 132 cases in the control group. The distribution and characteristics of gender, profession, and the segments of the lumbar disc about the two groups were shown in Table 1 . In comparison with the control group, patients with ULDH did not exhibit significant differences in age distribution and BMI distribution $(P>0.05)$. However, there were statistically significant differences $(P<0.05)$ in the TMCSA (Fig. 2A), PMCSA (Fig. 2B), AMMT (Fig. 2C), and the degree of fatty infiltration (Fig. 2D, E). The specific multifidus muscle measurement results were shown in Table 2 .
Table 1 Characteristics of study group and control group

\begin{tabular}{llll}
\hline Feature & Study group & Control group & $\boldsymbol{P}$ \\
\hline Age & & & $>0.05$ \\
$\quad$ Range & $47.47 \pm 10.918$ & $40.27 \pm 10.128$ & \\
$\quad$ Min - Med - Max & $21-51-64$ & $24-38.5-62$ & \\
BMI & & & \\
$<18.5$ & 4 & 10 & \\
$18.5 \sim 24.9$ & 68 & 86 & 1.000 \\
$\geq 25.0$ & 60 & 36 & \\
Gender & & & 1.000 \\
Male & 82 & 82 & \\
Female & 50 & 50 & \\
Profession & & & \\
Manual workers & 52 & 52 & \\
Non-manual laborer & 80 & 80 & \\
Segments & & & \\
L1/2 & 18 & 24 & \\
L2/3 & 24 & 90 & \\
L3/4 & 90 & & \\
\hline
\end{tabular}

BMI Body mass index

According to the multivariate logistic regression analysis of factors (Fig. 3), such as age, BMI, TMCSA, PMCSA, AMMW, and degree of fatty infiltration: the age $(P=0.251$, OR $0.966,95 \% \mathrm{CI} \quad 0.912-1.024)$ and $\operatorname{BMI}(P=0.136$, OR $1.135,95 \%$ CI $0.961-1.341)$ were not likely to be the impact factors for ULDH $(P>0.05)$; TMCSA $(P=0.023$, OR $0.980,95 \%$ CI $0.963-0.997)$, PMCSA $(P=0.014$, OR $0.973,95 \%$ CI $0.952-0.995)$, AMMW $(P=0.014$,OR $0.396,95 \%$ CI $0.189-0.830)$, degree of fatty infiltration (left: $P=0.004, \mathrm{OR} 1.585,95 \% \mathrm{CI}$ $1.155-2.175$; right: $P=0.012$, OR 1.459 , $95 \% \mathrm{CI} 1.087-$ 1.960) were possible impact factors for ULDH, and the differences were statistically significant $(P<0.05)$.

\section{Discussion}

The study showed that patients with ULDH exhibited degeneration of the multifidus muscle compared with the normal control group. It was demonstrated by the decreased cross-sectional area of the multifidus muscles and increased fatty infiltration. These findings agree with those from previous studies that investigated the association between lower lumbar degeneration and multifidus muscles [14, 21].

Back pain is the most common symptom of ULDH, and the resulting release of inflammatory factors may cause multifidus muscle degeneration and a decline in 


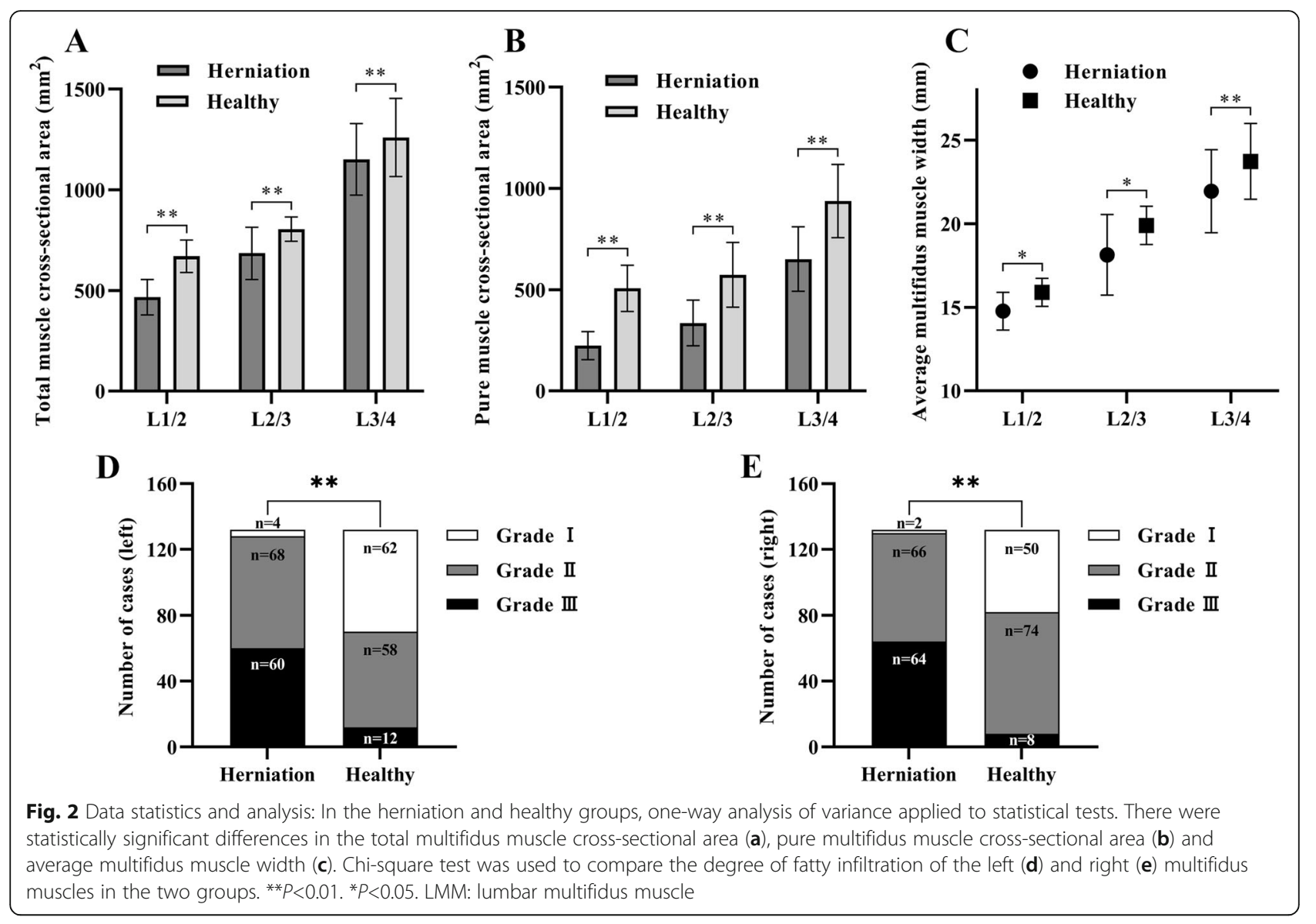

function. Meanwhile, the response mechanism of the human body that restricts lumbar activity to protect itself leads to further aggravation of the degeneration of the multifidus muscle. The multivariate logistic regression analysis confirmed that the degeneration of the

Table 2 Measurement results of the multifidus muscle

\begin{tabular}{lccc}
\hline Feature & Study group & Control group & $P$ \\
\hline TMCSA $\left(\mathrm{mm}^{2}\right)$ & & & $<0.05$ \\
L1/2 & $467.56 \pm 87.97$ & $670.78 \pm 80.27$ & \\
L2/3 & $685.25 \pm 129.32$ & $804.58 \pm 60.50$ & \\
L3/4 & $1152.11 \pm 178.10$ & $1260.91 \pm 193.89$ & \\
PMCSA $\left(\mathrm{mm}^{2}\right)$ & & & $<0.05$ \\
L1/2 & $223.89 \pm 69.09$ & $507.67 \pm 114.17$ & \\
L2/3 & $335.92 \pm 113.20$ & $574.00 \pm 159.66$ & \\
L3/4 & $652.36 \pm 158.82$ & $937.84 \pm 181.55$ & \\
AMMW (mm) & & & $<0.05$ \\
L1/2 & $14.78 \pm 1.13$ & $15.90 \pm 0.84$ & \\
L2/3 & $18.14 \pm 2.41$ & $19.90 \pm 1.15$ & \\
L3/4 & $21.95 \pm 2.49$ & $23.74 \pm 2.27$ & \\
\hline
\end{tabular}

TMCSA The total muscle cross-sectional area, PMCSA The pure muscle crosssectional area, $A M M W$ The average multifidus muscle width multifidus muscle and fatification of the multifidus muscle were the related factors of ULDH. Since this study is a retrospective study, it is difficult to establish the time sequence of the occurrence of multifidus muscle degeneration and ULDH so that the specific mechanism and relationship between both sides cannot be determined.

Studies related to the pathogenesis of multifidus muscle degeneration has revealed two main directions of study. Firstly, the analysis can consider changes to the size of muscle fibres from an anatomical and histological perspective. Kim et al. reported that in patients with $\mathrm{LDH}$, the cross-sectional area of the multifidus muscle decreased significantly, meanwhile the cross-sectional area of the psoas major muscle did not show any significant changes [22]. Reportedly, in patients with LDH, the average size of the type- 1 and type- 2 fibres of the ipsilateral multifidus muscles was significantly smaller than that of the contralateral side. This may be related to the fact that the innervation of the multifidus muscle on the ipsilateral side was by mono-segmental nerves, thus compression or damage to these nerve roots resulted in the atrophy of the type- 1 and type- 2 fibres [23]. An earlier study model of porcine nerve root injury discovered 


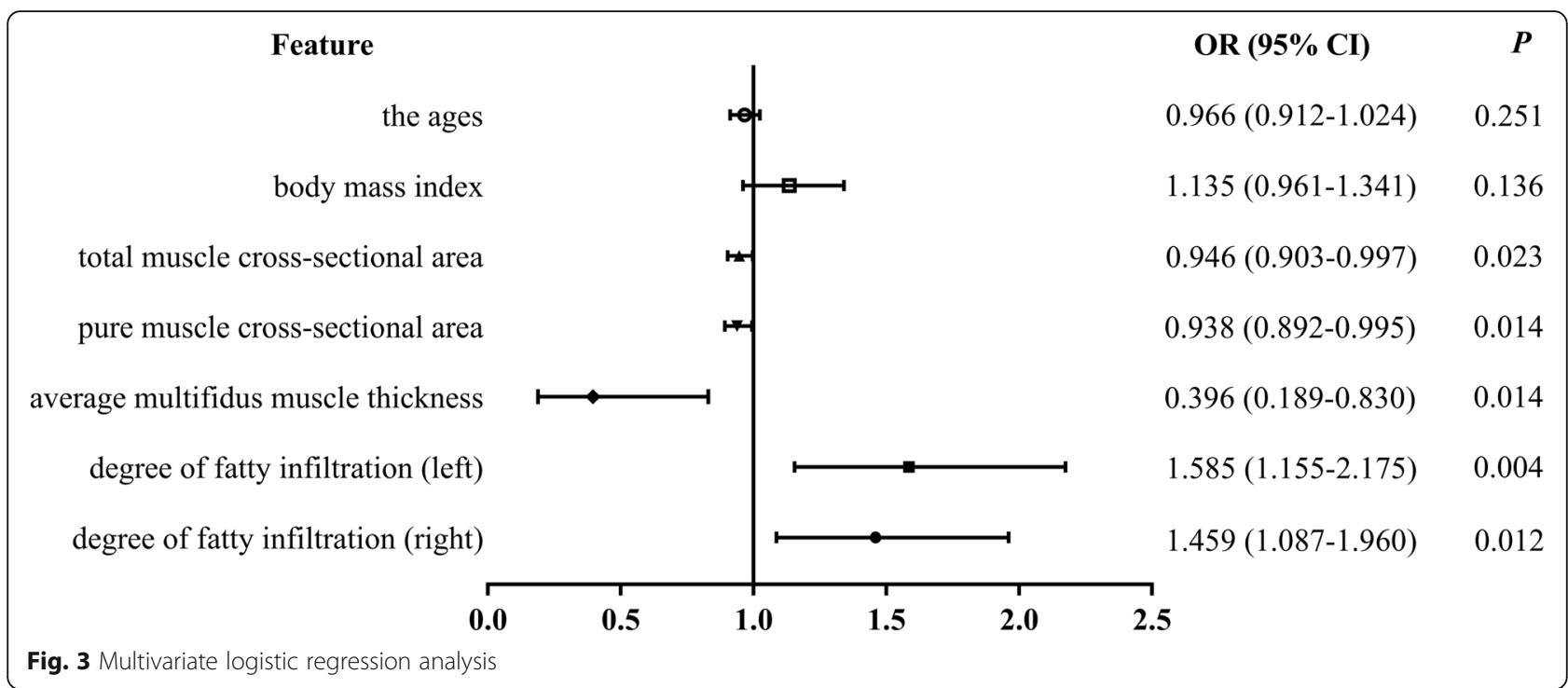

that the multifidus muscle atrophied rapidly with fatty infiltration following nerve root injury. Furthermore, the study also confirmed that, degeneration of the multifidus muscle after disc disease was usually confined to a single segment [24]. Yarjanian et al. used MRI to assess the difference in the cross-sectional area of the multifidus muscle among normal subjects, patients with low back pain, and those with LDH, the authors found that the extent of multifidus atrophy could not be elucidated by denervated innervation alone, since the atrophy was reversible [25].

Secondly, the analysis is performed through the crosssectional area and degree of fatty infiltration of the muscles from the morphological perspective. Faur et al. reported that multifidus muscle degeneration primarily occurred along the cross-section of MRI scans. Additionally, they reported that the degree of multifidus muscles degeneration was proportional to the degree of disc degeneration [26]. In this study, the patients with ULDH are significantly different from healthy people in the measurement indexes of the multifidus muscles. Moreover, the mean and range of relevant indicators are smaller. The results indicate that multifidus muscle atrophy is closely related to upper lumbar disc herniation.

Certain studies have reported that the degree of fatty infiltration of multifidus muscle at the same level as the ipsilateral segment of the LDH was significantly higher than on the contralateral segment. These findings confirmed the feasibility and reliability of visually evaluating the degree of fatty infiltration of multifidus muscle [6, 14, 17, 26]. Additionally, it was also reported that compared to assessing the cross-sectional area in patients with lumbar disc herniation, it was much more effective to assess the degree of multifidus fatty infiltration [27]. In the present study, both the cross-sectional area and the degree of fatty infiltration exhibited excellent evaluation efficiency.

Many clinical studies have confirmed the opinion that a correlation exists between multifidus muscle degeneration and LDH. However, the specific mechanism of the muscle degeneration remains unclear. Disuse and denervation are the two main mechanisms that are often mentioned [26]. Combined with the results of this study, the author assumes two models: On the one hand, degeneration of the multifidus muscles causes instability in the lumbar spine, which exacerbates degeneration of the upper lumbar intervertebral disc. On the other hand, the herniated lumbar disc presses on the nerve roots at the corresponding segment, which could lead to atrophy when the multifidus muscle is denervated.

Generally, denervation is believed to result in type-II fibre atrophy. However, there have been no unified reports on changes in type-I fibres [28]. Studies investigating the mechanism of disuse had reported a loss of functional exercise of the waist and back muscles, reduced capillary responsiveness, an insufficient supply of blood to the muscles, long-term hypoxia, and poor utilization of glycogen. The accumulation of metabolites resulted in muscle edema, and due to the disuse and degeneration of the muscles, the muscle tissues were gradually replaced by adipose tissues. It was believed that inflammatory or immunological injury mediated by inflammatory factors and injury to the psoas muscle caused by long-term heavy lumbar load degenerated the multifidus muscle $[28,29]$. In an extensive study that involved 2,028 cases, it was reported that multifidus muscle degeneration was more common in adult women and the severity was directly proportional to the age [30, 31]. Reportedly, the longer the symptoms lasted, the degeneration of the multifidus muscles were more severe 
[32]. Lumbosacral radiculopathy, reflex inhibition of discogenic lumbago, dorsal ramus syndrome, various genetic, and environmental as well as iatrogenic factors may lead to paravertebral muscle degeneration and fatty infiltration [26, 33]. Clinical practice had often neglected the paravertebral muscles, and it was not well protected during spinal surgeries in the past. This study also confirmed that there is significant atrophy of the multifidus muscle in patients with ULDH. Future clinical practise should emphasise on the negative effects of multifidus muscle atrophy. Reinforcing the lumbar spine muscles can help improve this atrophy, which could benefit the prevention and rehabilitation of ULDH.

Therefore, detailed researches studying the association between LMM and LDH and degenerative diseases require further attention. Having a complete understanding of this relationship could help to effectively strengthening the restoration of the normal physiological shape and function, and it could also benefit the prevention and rehabilitation of LDH [34-36]. The surgical approach should be optimized to minimize damage to surrounding tissue and maintain muscular integrity and neurovascular supply [37].

This study has some limitations. Firstly, the sample size is small, which may have increased sample error. Secondly, the specific background details of the sample may require additional in-depth research to further investigate the influencing factors of multifidus muscle degeneration. This study used visual difference of MRI signal intensity of different tissues for measurement and statistics, known as the "eyeballing method". However, this subjective visual assessment method has some limitations that cannot be ignored. Firstly, it is difficult to assess the interobserver variability. Secondly, performing drawings using the manual cursor technique could increase statistical errors. As a retrospective study, the precise mechanism and relationship between ULDH and multifidus muscle degeneration cannot be determined. Therefore, further research and analysis are needed.

\section{Conclusions}

A relationship could exist between multifidus muscles degeneration and ULDH entailing a process of mutual influence and interaction; however, prospective studies should confirm this. Evaluation of multifidus muscles should be considered when using MRI scans to assess the ULDH patients. Lumbar muscle strengthening training could prevent and improve muscle atrophy and degeneration.

\section{Abbreviations}

ULDH: Upper lumbar disc herniation; LDH: Lumbar disc herniation; CSA: Cross-sectional area; LMM: Lumbar multifidus muscle; MRI: Magnetic resonance imaging; PACS: Picture archiving and communication systems;
TMCSA: The total muscle cross-sectional area; PMCSA: The pure muscle cross-sectional area; AMMW: The average multifidus muscle width; BMI: Body mass index

\section{Acknowledgements \\ Not applicable.}

\section{Authors' contributions}

Study design and conception: XZ, CL, JX. Manuscript preparation: JX, CL. Imaging collection and measurement: $J, G M, T L$ and $H Z$. Statistical analysis: ZL, JX. Drawing of graphics and tables: ZZ, GX. Language editing and revision: AM, JX, CL. Final review and submission: $X Z$. All authors have read and approved the final manuscript.

\section{Funding}

This work was supported by the Youth Science Foundation of Guangxi Medical University, Grant/Award Numbers: GXMUYFY201712; Guangxi Young and Middle aged Teacher's Basic Ability Promoting Project, Grant/Award Number: 2019KY0119; National Natural Science Foundation of China, Grant/ Award Numbers: 81560359,81860393; The funder was not involved in designing the study or in the writing of this manuscript.

\section{Availability of data and materials}

The datasets used and/or analyzed during the current study are available from the corresponding author on reasonable request.

\section{Ethics approval and consent to participate}

This study was approved by the Ethics Committee of the First Affiliated Hospital of Guangxi Medical University, and obtained permissions to access clinical/personal patient data used in our research. All studies involving human participants were carried out in accordance with the ethical standards of the ethics committee and the Helsinki declaration. Written informed consent was obtained from all of the participants in the study.

Consent for publication

Not applicable.

\section{Competing interests}

The authors declare that they have no competing interests.

\section{Author details}

${ }^{1}$ Spine and Osteopathy Ward, The First Affiliated Hospital of Guangxi Medical University, No.6 Shuangyong Road, 530021 Nanning, Guangxi, People's Republic of China. ${ }^{2}$ Department of Orthopaedics, Southern Central Hospital of Yunnan Province (The First People's Hospital of Honghe State), Mengzi, Yunnan, People's Republic of China.

Received: 29 February 2020 Accepted: 11 January 2021

Published online: 19 January 2021

\section{References}

1. Benzakour T, Igoumenou V, Mavrogenis AF, Benzakour A. Current concepts for lumbar disc herniation. Int Orthopaed. 2019:43(4):841-51.

2. Sanderson SP, Houten J, Errico T, Forshaw D, Bauman J, Cooper PR. The unique characteristics of "upper" lumbar disc herniations. Neurosurgery. 2004;55(2):385-9.

3. Yüce I, Kahyaoğlu O, Mertan $P$, Çavuşoğlu H, Aydın Y. Analysis of clinical characteristics and surgical results of upper lumbar disc herniations. Neurochirurgie. 2019;65(4):158-63.

4. Kulig K, Scheid AR, Beauregard R, Popovich JM Jr, Beneck GJ, Colletti PM. Multifidus morphology in persons scheduled for single-level lumbar microdiscectomy: qualitative and quantitative assessment with anatomical correlates. Am J Phys Med Rehabil. 2009;88(5):355-61.

5. Battié MC, Niemelainen R, Gibbons LE, Dhillon S. Is level and side-specific multifidus asymmetry a marker for lumbar disc pathology? Spine J. 2012; 12(10):932-9.

6. Fortin M, Lazary A, Varga PP, McCall I, Battie MC. Paraspinal muscle asymmetry and fat infiltration in patients with symptomatic disc herniation. Eur Spine J. 2016;25(5):1452-9. 
7. Cholewicki J, Panjabi MM, Khachatryan A. Stabilizing function of trunk flexor-extensor muscles around a neutral spine posture. Spine (Phila Pa 1976). 1997;22(19):2207-12.

8. MacDonald DA, Moseley GL, Hodges PW. The lumbar multifidus: does the evidence support clinical beliefs? Man Ther. 2006;11(4):254-63.

9. Ward SR, Kim CW, Eng CM, Gottschalk LJt, Tomiya A, Garfin SR, et al. Architectural analysis and intraoperative measurements demonstrate the unique design of the multifidus muscle for lumbar spine stability. J Bone Joint Surg Am. 2009;91(1):176-85.

10. Panjabi MM. The stabilizing system of the spine. Part I. Function, dysfunction, adaptation, and enhancement. J Spinal Disord. 1992;5(4):383-9.

11. Macintosh JE, Bogduk N. The biomechanics of the lumbar multifidus. Clin Biomech (Bristol Avon). 1986;1(4):205-13.

12. Macintosh JE, Valencia F, Bogduk N, Munro RR. The morphology of the human lumbar multifidus. Clin Biomech (Bristol Avon). 1986;1(4):196-204.

13. Kalichman L, Carmeli E, Been E. The association between Imaging parameters of the paraspinal muscles, spinal degeneration, and low back pain. Biomed Res Int. 2017;2017:1-14.

14. Colakoglu B, Alis D. Evaluation of lumbar multifidus muscle in patients with lumbar disc herniation: are complex quantitative MRI measurements needed? J Int Med Res. 2019:47(8):3590-600.

15. Ploumis A, Michailidis N, Christodoulou P, Kalaitzoglou I, Gouvas G, Beris A. Ipsilateral atrophy of paraspinal and psoas muscle in unilateral back pain patients with monosegmental degenerative disc disease. Br J Radiol. 2011; 84(1004):709-13.

16. Kim K, Kim YH, Lee S. Investigation of optimal follower load path generated by trunk muscle coordination. J Biomech. 2011:44(8):1614-7.

17. Altinkaya N, Cekinmez M. Lumbar multifidus muscle changes in unilateral lumbar disc herniation using magnetic resonance imaging. Skeletal Radiol. 2016;45(1):73-7.

18. Kjaer P, Bendix T, Sorensen JS, Korsholm L, Leboeuf-Yde C. Are MRI-defined fat infiltrations in the multifidus muscles associated with low back pain? BMC Med. 2007;5:2-2.

19. Ranson CA, Burnett AF, Kerslake R, Batt ME, O'Sullivan PB. An investigation into the use of MR imaging to determine the functional cross sectional area of lumbar paraspinal muscles. Eur Spine J. 2006:15(6):764-73.

20. Battaglia PJ, Maeda Y, Welk A, Hough B, Kettner N. Reliability of the Goutallier classification in quantifying muscle fatty degeneration in the lumbar multifidus using magnetic resonance imaging. J Manipulative Physiol Ther. 2014;37(3):190-7.

21. Ekin EE, Kurtul Yıldız H, Mutlu H. Age and sex-based distribution of lumbar multifidus muscle atrophy and coexistence of disc hernia: an MRI study of 2028 patients. Diagn Interv Radiol. 2016a;22(3):273-6.

22. Kim WH, Lee SH, Lee DY. Changes in the cross-sectional area of multifidus and psoas in unilateral sciatica caused by lumbar disc herniation. J Korean Neurosurg Soc. 2011;50(3):201-4.

23. Yoshihara K, Shirai $Y$, Nakayama $Y$, Uesaka S. Histochemical changes in the multifidus muscle in patients with lumbar intervertebral disc herniation. Spine (Phila Pa 1976). 2001:26(6):622-6.

24. Hodges P, Holm AK, Hansson T, Holm S. Rapid atrophy of the lumbar multifidus follows experimental disc or nerve root injury. Spine (Phila Pa 1976). 2006;31(25):2926-33.

25. Yarjanian JA, Fetzer A, Yamakawa KS, Tong HC, Smuck M, Haig A Correlation of paraspinal atrophy and denervation in back pain and spinal stenosis relative to asymptomatic controls. Pm r. 2013:5(1):39-44.

26. Faur C, Patrascu JM, Haragus H, Anglitoiu B. Correlation between multifidus fatty atrophy and lumbar disc degeneration in low back pain. BMC Musculoskelet Disord. 2019;20(1):414-14

27. Bouche KG, Vanovermeire O, Stevens VK, Coorevits PL, Caemaert JJ, Cambier $D C$, et al. Computed tomographic analysis of the quality of trunk muscles in asymptomatic and symptomatic lumbar discectomy patients. BMC Musculoskelet Disord. 2011:12:65

28. Sun D, Liu P, Cheng J, Ma Z, Liu J, Qin T. Correlation between intervertebral disc degeneration, paraspinal muscle atrophy, and lumbar facet joints degeneration in patients with lumbar disc herniation. BMC Musculoskelet Disord. 2017;18(1):167-67.

29. Weber BR, Grob D, Dvorak J, Muntener M. Posterior surgical approach to the lumbar spine and its effect on the multifidus muscle. Spine (Phila Pa 1976). 1997;22(15):1765-72.

30. Ekin EE, Kurtul Yıldız H, Mutlu H. Age and sex-based distribution of lumbar multifidus muscle atrophy and coexistence of disc hernia: an MRI study of
2028 patients. Diagnostic and interventional radiology (Ankara. Turkey). 2016b:22(3):273-6.

31. Johannesdottir F, Allaire B, Anderson DE, Samelson EJ, Kiel DP, Bouxsein ML. Population-based study of age- and sex-related differences in muscle density and size in thoracic and lumbar spine: the Framingham study. Osteoporos Int. 2018;29(7):1569-80.

32. Chen ZN, Yao XM, LV Y, He BJ, Ye JC, Shao RX, et al. Morphology of the lumbar multifidus muscle in lumbar disc herniation at different durations and at different ages. Exp Ther Med. 2018;15(5):4119-26.

33. Kang J-I, Kim S-Y, Kim J-H, Bang H, Lee I-S. The location of multifidus atrophy in patients with a single level, unilateral lumbar radiculopathy. Ann Rehabil Med. 2013;37(4):498-504

34. Choi G, Raiturker PP, Kim MJ, Chung DJ, Chae YS, Lee SH. The effect of early isolated lumbar extension exercise program for patients with herniated disc undergoing lumbar discectomy. Neurosurgery. 2005:57(4):764-72.

35. Woodham M, Woodham A, Skeate JG, Freeman M. Long-term lumbar multifidus muscle atrophy changes documented with magnetic resonance imaging: a case series. J Radiol Case Rep. 2014;8(5):27-34

36. Hiyama A, Katoh H, Sakai D, Tanaka M, Sato M, Watanabe M. The correlation analysis between sagittal alignment and cross-sectional area of paraspinal muscle in patients with lumbar spinal stenosis and degenerative spondylolisthesis. BMC Musculoskelet Disord. 2019;20(1):352-52.

37. Deng $X$, Zhu Y, Wang S, Zhang Y, Han H, Zheng D, et al. CT and MRI determination of intermuscular space within lumbar paraspinal muscles at different intervertebral disc levels. PloS one. 2015;10(10):e0140315-e15.

\section{Publisher's Note}

Springer Nature remains neutral with regard to jurisdictional claims in published maps and institutional affiliations.
Ready to submit your research? Choose BMC and benefit from:

- fast, convenient online submission

- thorough peer review by experienced researchers in your field

- rapid publication on acceptance

- support for research data, including large and complex data types

- gold Open Access which fosters wider collaboration and increased citations

- maximum visibility for your research: over $100 \mathrm{M}$ website views per year

At BMC, research is always in progress.

Learn more biomedcentral.com/submissions 